\title{
ON THE COMPOSITION OF FIELDS
}

\author{
CLAUDE CHEVALLEY
}

Let $K / k, K^{\prime} / k$ be two extensions of a basic field $k$. By a composite extension of these two extensions, we understand the complex notion formed of an extension $\Omega / k$ of $k$, of an isomorphism $\tau$ of $K / k$ into $\Re / k$ and of an isomorphism $\tau^{\prime}$ of $K^{\prime} / k$ into $\Re / k$, provided the following conditions are verified:

(1) $\Re$ is generated by the two fields $K^{\tau}, K^{\prime \tau^{\prime}}$.

(2) If $A, A^{\prime}$ are subsets of $K, K^{\prime}$ respectively which are algebraically independent over $k$, the set $A^{\tau} \cup\left(A^{\prime}\right)^{\tau^{\prime}}$ is algebraically independent over $k$. In other words, the algebraic relations which hold in $\Re$ between elements of $K^{\tau}, K^{\prime \tau^{\prime}}$ are consequences of the algebraic relations which hold between elements of $K^{\tau}$ alone or of $K^{\prime \tau^{\prime}}$ alone. ${ }^{1}$

Theorem 1. Any two given extensions $K / k, K^{\prime} / k$ have at least one composite extension.

Let $B^{\prime}$ be a transcendence basis for $K^{\prime} / k$. We can find a purely transcendental extension $\Omega / K$ which has a transcendence basis $B^{\prime \tau^{\prime}}$ with the same cardinal number as $B^{\prime}\left(\tau^{\prime}\right.$ stands for a one-to-one mapping of $B^{\prime}$ onto $\left.B^{\prime \tau^{\prime}}\right)$. The algebraic closure $\bar{\Omega}$ of $\Omega$ contains the algebraic closure $\overline{\mathrm{P}}$ of the field $\mathrm{P}=k\left(B^{\prime \tau^{\prime}}\right)$. The mapping $\tau^{\prime}$ may be extended to an isomorphism of $K^{\prime} / k$ with an extension $K^{\prime \tau^{\prime}} / k$ contained in $\overline{\mathrm{P}} / k$, and a fortiori in $\bar{\Omega}$. We set $\Re=K K^{\prime \tau^{\prime}}$, and denote by $\tau$ the identity mapping of $K / k$ into $\Omega / k$. We claim that the system $\left(\Omega / k, \tau, \tau^{\prime}\right)$ is a composite extension of $K / k, K^{\prime} / k$.

It is sufficient to check the condition (2), and we may assume without loss of generality that $A, A^{\prime}$ are finite. There exists a finite subset $B_{1}^{\prime}$ of $B^{\prime}$ such that $k\left(A^{\prime}, B_{1}^{\prime}\right)$ is algebraic over $k\left(B_{1}^{\prime}\right)$. Let $d, d^{\prime}, e$ be the number of elements in $A, A_{1}^{\prime}, B_{1}^{\prime}$. The elements of $B_{1}^{\prime}$, being algebraically independent over $K$, are a fortiori algebraically independent over $k(A)$. Therefore, the degree of transcendency of $k\left(A, A^{\prime \tau^{\prime}}, B_{1}^{\prime} \tau^{\prime}\right)$ over $k$ is $d+e$. The degree of transcendency of $k\left(A_{1}^{\prime} \tau^{\prime}, B_{1}^{\prime} \tau^{\prime}\right)$ over $k\left(A_{1}^{\prime} \tau^{\prime}\right)$ is $e-d^{\prime}$. The degree of transcendency $f$ of $k\left(A, A^{\prime \tau^{\prime}}, B_{1}^{\prime} \tau^{\prime}\right)$ over $k(A)$ is therefore less than or equal to $e-d$. It follows that the degree of transcendency of $k\left(A, A^{\prime \tau^{\prime}}\right)$ over $k$, which

Received by the editors October 1, 1941.

1 The problem of composite extensions has been considered by Zariski (Algebraic varieties over ground fields of characteristic zero, American Journal of Mathematics, vol. 62 (1940), pp. 187-221) in the case when one of the extensions $K / k, K^{\prime} / k$ is algebraic and normal. 
is $d+e-f$, is at least equal to $d+d^{\prime}$, which proves that $A \cup A^{\prime \tau^{\prime}}$ is algebraically independent over $k$.

Let $\left(\Omega / k, \tau, \tau^{\prime}\right)$ and $\left(\Omega_{1} / k, \tau_{1}, \tau_{1}^{\prime}\right)$ be two composite extensions of $K / k, K^{\prime} / k$. We shall say that these extensions are isomorphic if there exists an isomorphism $\sigma$ of $\Omega / k$ with $\Omega^{\prime} / k$ such that $\tau_{1}=\sigma \tau, \tau_{1}^{\prime}=\sigma \tau^{\prime}$.

The consideration of the case where $K / k, K^{\prime} / k$ are algebraic over $k$ (but not normal) shows immediately that there are in general several non-isomorphic types of composite extensions. The extreme opposite case occurs when $k$ is algebraically closed in $K$ and $K^{\prime}$ ( $k$ is said to be algebraically closed in $K$ if every element of $K$ which is algebraic over $k$ lies already in $k$ ). In that case, the composite extension $\Omega / k$ turns out to be unique; but, unfortunately, $k$ may fail to be algebraically closed in $\Omega$. For instance, let us take $K=K^{\prime}=k\left(x,\left(a+b x^{p}\right)^{1 / p}\right)$, where $p \neq 0$ is the characteristic of $k$, and where $a, b$ are elements of $k$ such that $k\left((a)^{1 / p},(b)^{1 / p}\right)$ is of degree $p^{2}$ over $k$. It is easy to verify that $k$ is algebraically closed in $K$; on the other hand, the composite extension is $k\left(x, y,\left(a+b x^{p}\right)^{1 / p},\left(a+b y^{p}\right)^{1 / p}\right) / k=k\left(x, y,(a)^{1 / p},(b)^{1 / p}\right) / k$.

We shall get around this difficulty by introducing the following notion:

Definition 1. A field $k$ is said to be quasi-algebraically closed (q.a.c.) in $K$ if every element a of $K$ which is algebraic over $k$ is purely inseparable over $k$ (that is, is the unique root of some equation with coefficients in $k)$.

We shall prove the following theorem:

TheOREm 2. Let $\left(\Omega / k, \tau, \tau^{\prime}\right)$ and $\left(\Omega_{1} / k, \tau_{1}, \tau_{1}^{\prime}\right)$ be two composite extensions of the extensions $K / k, K^{\prime} / k$. Let $L, L^{\prime}$ be fields such that: (1) $k \subset L \subset K, k \subset L^{\prime} \subset K^{\prime}$; (2) $L, L^{\prime}$ are algebraic over $k$; (3) $L$ is q.a.c. in $K$ and $L^{\prime}$ is q.a.c. in $K^{\prime}$. If an isomorphism $\sigma_{0}$ of $L^{\tau} L^{\prime \tau^{\prime}} / k$ with $L^{\tau_{1}} L^{\prime \tau_{1}^{\prime}} / k$ is such that $\sigma_{0} \tau$ coincides on $L$ with $\tau_{1}$ and $\sigma_{0} \tau^{\prime}$ coincides on $L^{\prime}$ with $\tau_{1}^{\prime}$, then $\sigma_{0}$ may be extended to an isomorphism of $\left(\Omega / k, \tau, \tau^{\prime}\right)$ with $\left(\Omega_{1} / k, \tau_{1}, \tau_{1}^{\prime}\right)$.

In other words, the type of the composite extension $\left(\Omega / k, \tau, \tau^{\prime}\right)$ is determined by the type of the composite extension $\left(L^{\tau} L^{\prime \tau^{\prime}} / k, \tau, \tau^{\prime}\right)$ of $L / k, L^{\prime} / k$.

We shall first prove three lemmas. The fields which are considered in the first two of these lemmas are assumed to be all subfields of some all-inclusive field.

LEMMA 1. Let $k$ be a q.a.c. sub-field of a field $K$. Let $Z$ be a field such that $k \subset Z \subset K$, and $y$ be an element of $K$ which is a root of an irreducible equation $F=0$ with coefficients in $Z$. Let $\Omega / k$ be an algebraic extension 
of $k, \alpha$ be an element of $\Omega$ and $\Phi=0$, the irreducible equation in $k$ which is satisfied by $\alpha$. Then the polynomial $F$ is a power of an irreducible polynomial in the field $\Omega Z$, and $\Phi$ is a power of an irreducible polynomial in $K$. Moreover, $\Omega$ is q.a.c. in the field $K \Omega$.

We may assume without loss of generality that $\Omega=k(\alpha)$. Let $L$ be the algebraic closure of $k$ in $K$ (that is, the field consisting of the elements of $K$ which are algebraic over $k$ ). Let $\Phi_{1}=0$ be the irreducible equation satisfied by $\alpha$ in $L$. Therefore $\Phi_{1}$ divides $\Phi$. On the other hand, if $p$ is the characteristic of $k$, the polynomial $\Phi_{1}^{p^{u}}$ has its coeffcients in $k$ if $u$ is large enough, and therefore $\Phi$ divides $\Phi_{1}^{p^{u}}$. It follows that $\Phi$ is a power of $\Phi_{1}$.

If $\Phi_{1}^{\prime}=0$ is the irreducible equation satisfied by $\alpha$ in $K$ then $\Phi_{1}^{\prime}$ divides $\Phi_{1}$. Therefore, every root of the equation $\Phi_{1}^{\prime}=0$ is also a root of $\Phi_{1}=0$, which shows that the coefficients of $\Phi_{1}^{\prime}$ are algebraic over $k$. It follows that $\Phi_{1}^{\prime}=\Phi_{1}$ which proves that $\Phi$ is a power of an irreducible polynomial in $K$.

Let $F_{1}=0$ be the irreducible equation satisfied by $y$ in $L Z$, and let $m$ be the degree of $F_{1}$. If $n$ is the degree of $\phi_{1}$, we have

$$
[L Z(y, \alpha): L Z]=[L Z(y, \alpha): L Z(y)][L Z(y): L Z]=m n
$$

because $\Phi_{1}$ is irreducible in $K$, and a fortiori, in $L Z(y)$. It follows that

$$
[L Z(y, \alpha): L Z(\alpha)][L Z(\alpha): L Z]=m n .
$$

But $[L Z(\alpha): L Z]=n$, since $\Phi_{1}$ is irreducible in $L Z$; therefore we have $[L Z(y, \alpha): L Z(\alpha)]=n$ which shows that $F_{1}$ is irreducible in $L Z(\alpha)$. On the other hand, $F_{1}$ divides $F$; since $L Z$ is purely inseparable over $Z$, the same argument which was used above for $\Phi_{1}$ shows that $F$ is a power of $F_{1}$. Since $Z \subset \Omega Z=Z(\alpha) \subset L Z(\alpha), F$ is also a power of an irreducible polynomial in $\Omega Z$.

There remains to prove that $k(\alpha)$ is q.a.c. in $K(\alpha)$. Let $\beta$ be an element of $K(\alpha)$ which is algebraic over $k(\alpha)$, and therefore also on $k$. There exists a power $\alpha^{p s}=\alpha_{1}$ of $\alpha$ which is separable over $k$; we set $\beta_{1}=\beta^{p s}$, whence $\beta_{1} \in K\left(\alpha_{1}\right)$ and

$$
\beta_{1}=\xi_{0}+\xi_{1} \alpha_{1}+\cdots+\xi_{h-1} \alpha_{1}^{h-1}, \quad \xi_{i} \in K,
$$

where $h=\left[K\left(\alpha_{1}\right): K\right]$. If we write the corresponding formulas for the conjugates of $\beta_{1}$ with respect to $K$, and observe that $\alpha_{1}$ is different from its conjugates, we see that the $\xi_{i}$ 's may be expressed rationally by means of the conjugates of $\alpha_{1}, \beta_{1}$. It follows that $\xi_{0}, \xi_{1}, \cdots, \xi_{h-1}$ are algebraic over $k$, and therefore belong to $L$. Since $L$ is purely in- 
separable over $k$, we may conclude that some power $\beta_{1}^{p^{t}}$ of $\beta_{1}$ lies in $k\left(\alpha_{1}\right)$, where $p$ is the characteristic of $k\left(\beta_{1} \in k\left(\alpha_{1}\right)\right)$ (if $\left.p=0\right)$. We have $\beta^{p^{s+t}} \in \Omega$ which completes the proof of the lemma.

Before stating Lemma 2, we have to introduce another notion. Two extensions $K / k, \Omega / k$ of the field $k$ which are contained in some larger field are said to be algebraically dissociated if the following condition is realized: If $A, B$ are subsets of $K, \Omega$, respectively, which are algebraically independent over $k$, the set $A \cup B$ is also algebraically independent.

LEMMA 2. The Lemma 1 (abstraction made of what concerns $\alpha$ and $\Phi$ ) remains valid when $\Omega / k$ is any extension of $k$, provided the extensions $\Omega / k, K / k$ are algebraically dissociated.

Let $C$ be a transcendence basis of $\Omega / k$ and $\Omega_{0}$ the field $k(C)$. Under our assumption the extension $K \Omega_{0} / K$ is purely transcendental. We claim that $\Omega_{0}$ is q.a.c. in $K \Omega_{0}$. It will of course be sufficient to prove it in the case where $C$ consists $^{2}$ in a single element $t$. Let $P(t) / Q(t)=\omega$ be an element of $K \Omega_{0}=K(t)$ (where $P(t), Q(t)$ are polynomials with coefficients in $K$ ). We shall prove that if $\omega$ is algebraic over $\Omega_{0}$, it can be expressed as a rational function in $t$ with coefficients in $L$ (the algebraic closure of $k$ in $K$ ). From this result it will follow that $L \Omega_{0}$ is algebraically closed in $K \Omega_{0}$, and therefore that $\Omega_{0}$ is q.a.c. in $K \Omega_{0}$.

The proof will proceed by induction on the number $l=d^{\circ} P+d^{\circ} Q$ where $d^{\circ} P, d^{\circ} Q$ denote the degrees of $P, Q$ with respect to $t$. It is obvious for $l=0$; assume that the result holds for $l-1$. If either one of the elements $P(0), Q(0)$ is null, we can reduce ourselves to the case $l-1$ by considering instead of $\omega$ one of the elements $\omega / t, t \omega$ (these elements are also algebraic over $\Omega_{0}$ ). So, let us assume that $P(0) Q(0) \neq 0$. We have by assumption a relation of the form

$$
A_{0}(t) P^{n}(t)+A_{1}(t) P^{n-1}(t) Q(t)+\cdots+A_{n}(t) Q^{n}(t)=0,
$$

where $A_{0}(t), \cdots, A_{n}(t)$ are polynomials in $t$ with coefficients in $k$, not all divisible by $t$. Putting $t=0$, we conclude that $P(0) / Q(0)$ is algebraic over $k$, and therefore belongs to $L$. The element $\omega^{\prime}=P(t) / Q(t)$ $-P(0) / Q(0)$ is again algebraic over $\Omega_{0}$ and may be written in the form $t P^{\prime}(t) / Q^{\prime}(t)$ with $d^{\circ} P^{\prime}+d^{\circ} Q^{\prime}=l-1$. Therefore $\omega^{\prime} / t \in L(t)$ and $\omega \in L(t)$, which proves our assertion.

The extension $Z \Omega_{0} / Z$ being purely transcendental (because $K \Omega_{0} / K$ is), the polynomial $F$, which is irreducible in $Z$, remains irreducible

2 This property was proved in the paper, Pencils on an algebraic variety and a new proof of a theorem of Bertini, by Zariski, Transactions of this Society, vol. 50 (1941), pp. $48-70$. 
in $Z \Omega_{0}$. The extension $\Omega / \Omega_{0}$ being algebraic, it follows from Lemma 1 (applied with $\Omega_{0}$ instead of $k$ ) that $F$ becomes a power of an irreducible polynomial in $Z \Omega$ and that $\Omega$ is q.a.c. in $K \Omega$; Lemma 2 has been proved.

We pass now to the third lemma. The notations used in this lemma are the same as those introduced in the statement of Theorem 2 .

Lemma 3. Let $Z$ be a field such that $L \subset Z \subset K$, and let $y$ be an element of $K$. (1) If $y$ is transcendental over $Z, y^{\tau}$ is transcendental over $Z^{\tau} K^{\prime \tau^{\prime}}$. (2) If $y$ is a root of the irreducible equation $F=0$ in $Z$, the polynomial $F^{r}$ is a power of an irreducible polynomial in $Z^{\tau} K^{\prime \tau^{\prime}}$.

(1) Let $B^{\prime}$ be a transcendence basis of $K^{\prime} / k$ and $C$ be a transcendence basis of $Z / k$; hence $C^{\tau} \cup B^{\prime \tau^{\prime}}$ is a transcendence basis of $Z^{\tau} K^{\prime \tau^{\prime}} / k$. If $y$ is transcendental over $Z$, the set $C \cup\{y\}$ is algebraically independent; therefore $C^{\tau} \cup B^{\prime \tau^{\prime}} \cup\left\{y^{\tau}\right\}$ is algebraically independent over $k$, which proves that $y^{\tau}$ is transcendental over $Z^{\tau} K^{\prime \tau^{\prime}}$.

(2) Since the extensions $Z^{r} / k, K^{\prime r^{\prime}} / k$ are clearly algebraically dissociated over $k$, assertion (2) results from Lemma 2.

We pass now to the proof of Theorem 2 . We consider the set $\Sigma$ of the systems $\left(Z, Z^{\prime}, \sigma\left(Z, Z^{\prime}\right)\right)$ composed (a) of fields $Z, Z^{\prime}$ such that $L \subset Z \subset K, L^{\prime} \subset Z^{\prime} \subset K^{\prime}$; (b) of an isomorphism $\sigma\left(Z, Z^{\prime}\right)$ of $Z^{r} Z^{\prime \tau^{\prime}}$ with $Z^{r_{1}} Z^{\prime \tau_{1}^{\prime}}$ such that $\sigma\left(Z, Z^{\prime}\right) \tau$ coincides with $\tau_{1}$ on $Z$, that $\sigma\left(Z, Z^{\prime}\right) \tau^{\prime}$ cocincides with $\tau_{1}^{\prime}$ on $Z^{\prime}$, and that $\sigma\left(Z, Z^{\prime}\right)$ coincides with $\sigma_{0}$ on $L^{\tau} L^{\prime \tau^{\prime}}$. We order the set $\Sigma$ by the convention that

$$
\left(Z, Z^{\prime}, \sigma\left(Z, Z^{\prime}\right)\right) \leqq\left(U, U^{\prime}, \sigma\left(U, U^{\prime}\right)\right)
$$

if $Z \subset U, Z^{\prime} \subset U^{\prime}$ and $\sigma\left(U, U^{\prime}\right)$ coincides with $\sigma\left(Z, Z^{\prime}\right)$ on $Z^{r} Z^{\prime \tau^{\prime}}$. It is trivial to verify that in this ordered set every completely ordered subset has an upper bound. Hence, by Zorn's theorem, $\Sigma$ has a maximal element, which we denote from now on by $\left(Z, Z^{\prime}, \sigma\right)$. Theorem 2 will be proved if we can show that $Z=K, Z^{\prime}=K^{\prime}$.

Let $y$ be an element of $K$, and assume for a moment that $y$ is transcendental over $Z$. Then $y^{\tau}$ is transcendental over $Z^{\tau}$ and $y^{\tau 1}$ is transcendental over $Z^{r_{1}}$. By Lemma 3, $y$ is also transcendental over $Z^{\tau} Z^{\prime \tau^{\prime}}$, and a fortiori, over $Z^{\tau} Z^{\prime \tau^{\prime}}$. Similarly, $y^{\tau_{1}}$ is transcendental over $Z^{r_{1}} Z^{\prime \tau_{1}{ }^{\prime}}$. We set $U=Z(y)$; then it is possible to extend $\sigma$ to an isomorphism $\sigma^{*}$ of $U^{r} Z^{\prime \tau^{\prime}}$ with $U^{r_{1}} Z^{\prime \tau_{1}{ }^{\prime}}$ in such a way that $\sigma^{*}\left(y^{\tau}\right)=y^{\tau_{1}}$. It follows that $\sigma^{*} \tau$ coincides with $\tau_{1}$ on $U$ and that $\sigma^{*} \tau^{\prime}$ coincides with $\tau_{1}^{\prime}$ on $Z^{\prime}$. But this is contrary to the maximality of $\left(Z, Z^{\prime}, \sigma\right)$.

It follows that $K$ is algebraic over $Z$, and similarly that $K^{\prime}$ is algebraic over $Z^{\prime}$.

Let us again consider the element $y \in K$; it is a root of an irreducible 
equation $F=0$ in $Z$. By Lemma $3, F^{\tau}$ becomes in $Z^{\tau} Z^{\prime \tau^{\prime}}$ a power of an irreducible polynomial $F_{1}$, and we have $F_{1}\left(y^{\tau}\right)=0$. The polynomial

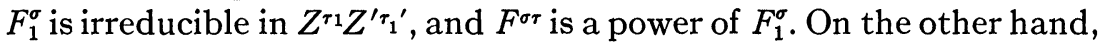
we have $F^{\sigma \tau}=F^{\tau_{1}}$ and $F^{\tau_{1}}\left(y^{\tau_{1}}\right)=0$, whence $F_{1}^{\sigma}\left(y^{\tau_{1}}\right)=0$. Therefore, we may extend $\sigma$ to an isomorphism $\sigma^{*}$ of $Z^{\tau} Z^{\prime \tau^{\prime}}\left(y^{\tau}\right)$ with $Z^{\tau_{1}} Z^{\prime \tau_{1}}\left(y^{\tau_{1}}\right)$ such that $\sigma^{*}\left(y^{\tau}\right)=y^{\tau_{1}}$. The isomorphism $\sigma^{*} \tau$ of $Z(y)$ into $K^{r_{1}} K^{\prime \tau_{1}{ }^{\prime}}$ coincides with the automorphism induced by $\tau_{1}$ and $\sigma^{*} \tau^{\prime}$ coincides on $Z^{\prime}$ with $\tau_{1}^{\prime}$. By the maximality property of $\left(Z, Z^{\prime}, \sigma\right)$, we have $Z(y)=Z$, whence $K=Z$, and we see in the same way that $K^{\prime}=Z^{\prime}$, which completes the proof of Theorem 2 .

COROLlary. Let $K / k, K^{\prime} / k$ be two extensions of $k$, and assume that $k$ is q.a.c. in at least one of them. Then there exists only one type of composite extension of our two extensions.

In fact, if $k$ is q.a.c. in $K^{\prime}$, we may apply Theorem 2 with $L^{\prime}=k^{\prime}$. If we set $\sigma_{0}=\tau_{1} \tau^{-1}, \sigma_{0}$ is an isomorphism of $L^{r}$ with $L^{\tau_{1}}$ and $\sigma_{0} \tau$ coincides with $\tau_{1}$ on $L$; it follows that $\sigma_{0}$ may be extended to an isomorphism of $\left(\Omega, \tau, \tau^{\prime}\right)$ with $\left(\Omega_{1}, \tau_{1}, \tau_{1}^{\prime}\right)$.

TheOREM 3. Let $\left(\Re / k, \tau, \tau^{\prime}\right)$ be a composite extension of $K / k, K^{\prime} / k$ and let $L, L^{\prime}$ be fields which satisfy the conditions (1), (2), (3) of Theorem 2. Then $L^{\tau} L^{\prime \tau^{\prime}}$ is q.a.c. in $\Omega$.

By Lemma $1, L^{\tau} L^{\prime \tau^{\prime}}$ is q.a.c. in $L^{\tau} K^{\tau^{\prime}}$; by Lemma 2, we see that $L^{\tau} K^{\prime \tau^{\prime}}$ is q.a.c. in $K^{\tau} K^{\prime \tau^{\prime}}$, because the extensions $K^{\tau} / k, K^{\prime \tau^{\prime}} / k$ are algebraically dissociated. Let $\alpha$ be an element of $\Omega$ which is algebraic over $L^{\tau} L^{\prime \tau^{\prime}}$; then, if $p$ is the characteristic of $k$, the second result shows that, for $s$ large enough, $\alpha^{p^{s}} \in L^{\tau} K^{\prime \tau^{\prime}}$. Since $\alpha^{p^{s}}$ is also algebraic over $L^{\tau} L^{\prime \tau^{\prime}}$, the first result shows that $\left(\alpha^{p^{s}}\right)^{p^{p^{\prime}}}=\alpha^{p^{s+8^{\prime}}} \in L^{\tau} L^{\prime \tau^{\prime}}$, for $s^{\prime}$ large enough, which proves Theorem 3.

Corollary. If $k$ is q.a.c. in both $K$ and $K^{\prime}$, it is also q.a.c. in $K^{r} K^{\prime \tau^{\prime}}$.

Princeton University 